\title{
A TEMPERATURE DEPENDENT
}

\section{D-ACAR STUDY OF UNTWINNED METALLIC YBa $\mathrm{Cu}_{3} \mathrm{O}_{7-\mathrm{x}}{ }^{*}$}

\author{
L.C. Smedskjaer, ${ }^{1}$ A. Bansil, ${ }^{3}$ U. Welp, ${ }^{2}$ Y. Fang, ${ }^{1}$ and K.G. Bailey ${ }^{1}$ \\ 1Materials Science Division \\ and \\ 2Science \& Technology Center for Superconductivity \\ Argonne National Laboratory, Argonne, IL 60439
}

3Physics Department, Northeastern University, Boston, M1A 02115

The submitted manuscript has been authored by
a contractor of the U.S. Govemment under
contract No. W-3t-109-ENG-38. Accordingly,
the U.S. Govemment retains a nonexclusive,
royalty-free license to publish or reproduce the
published form of this contribution, or allow
cthers to do so, for U.S. Government pumoses.

jmc

\begin{abstract}
DISCLAIMER
This report was prepared as an account of work sponsored by an agency of the United States Government. Neither the United States Government nor any agency thereof, nor any of their employees, makes any warranty, express or implied, or assumes any legal liability or responsibility for the accuracy, completeness, or usefulness of any information, apparatus, product, or process disclosed, or represents that its use would not infringe privately owned rights. Reference herein to any specific commercial product, process, or service by trade name, trademark, manufacturer, or otherwise does not necessarily constitute or imply its endorsement, recommendation, or favoring by the United States Government or any agency thereof. The views and opinions of authors expressed herein do not necessarily state or reflect those of the United States Government or any agency thereof.
\end{abstract}

\footnotetext{
*Work supported by the U.S. Department of Energy, BES-Materials Sciences under contract \#W-31-109-ENG-38 (LC, YF, KGB) and the National Science Foundation Office of Science and Technology Centers under contract \#STC-8809854 (UW).
}

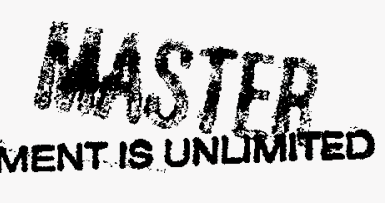




\section{DISCLAIMER}

Portions of this document may be illegible in electronic image products. Images are produced from the best available original document. 


\title{
A TEMPERATURE DEPENDENT 2D-ACAR STUDY OF UNTWINNED METALLIC $\mathrm{YBa}_{2} \mathrm{Cu}_{3} \mathrm{O}_{7-\mathrm{x}}$
}

\author{
L.C. Smedskjaer ${ }^{1}$, A. Bansil ${ }^{3}$, U. Welp ${ }^{2}$, Y. Fang1 ${ }^{1}$, and K.G. Bailey1 . \\ 1) Materials Science Division and 2) Science and Technology Center for Superconductivity. \\ Argonne National Laboratory, Argonne, II 60439. \\ 3) Physics Department, Northeastern University, Boston, Massachusetts 02115
}

\begin{abstract}
We have carried out 2D-ACAR measurements in the c-axis projection on an untwinned single crystal of $\mathrm{YBa}_{2} \mathrm{Cu}_{3} \mathrm{O}_{7-\mathrm{x}}$ as a function of temperature, for five temperatures ranging from $30 \mathrm{~K}$ to $300 \mathrm{~K}$. These temperature dependent $2 \mathrm{D}$-ACAR spectra can be approximated by a superposition of two temperature independent spectra with temperature dependent weighting factors. We discuss how the temperature dependence of the data can be exploited to obtain a 'background corrected' experimental spectrum, which is found to be in remarkable accord with the corresponding band theory based predictions, including for the first time the overall amplitude of the anisotropy in the 2D-ACAR. The corrected data also shows clear signatures of the ridge Fermi surface and an indication of the pillbox surface.
\end{abstract}

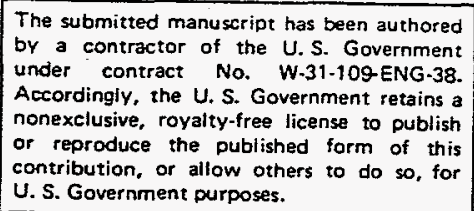




\title{
A TEMPERATURE DEPENDENT 2D-ACAR STUDY OF UNTWINNED METALLIC YBa2 $\mathrm{Cu}_{3} \mathrm{O}_{7-x}$
}

L.C. Smedskjaer ${ }^{1}$, A. Bansil ${ }^{3}$, U. Welp ${ }^{2}$, Y. Fang ${ }^{1}$, and K.G. Bailey1.

1) Materials Science Division and 2) Science and Technology Center for Superconductivity. Argonne National Laboratory, Argonne, II 60439.

3) Physics Department, Northeastern University, Boston, Massachusetts 02115

\begin{abstract}
We have carried out 2D-ACAR measurements in the $\mathrm{c}$-axis projection on an untwinned single crystal of $\mathrm{YBa}_{2} \mathrm{Cu}_{3} \mathrm{O}_{7-\mathrm{x}}$ as a function of temperature, for five temperatures ranging from $30 \mathrm{~K}$ to $300 \mathrm{~K}$. These temperature dependent $2 \mathrm{D}-\mathrm{ACAR}$ spectra can be approximated by a superposition of two temperature independent spectra with temperature dependent weighting factors. We discuss how the temperature dependence of the data can be exploited to obtain a 'background corrected' experimental spectrum, which is found to be in remarkable accord with the corresponding band theory based predictions, including for the first time the overall amplitude of the anisotropy in the 2D-ACAR. The corrected data also shows clear signatures of the ridge Fermi surface and an indication of the pillbox surface.
\end{abstract}

\section{Introduction}

Positron annihilation (2D-ACAR) studies of $\mathrm{YBa}_{2} \mathrm{Cu}_{3} \mathrm{O}_{7-x}$ started immediately after the discovery of this archetypal high temperature superconductor. The 2D-ACAR experiment is ideally suited to answer one of the fundamental questions that arose early, namely, whether the electron momentum density in the new materials possesses Fermi surface breaks like the conventional metals. Results in this regard would provide guidance in searching for a suitable theoretical description of the electronic structure, and thus help in developing an understanding of the mechanism responsible for the occurrence of high superconducting transition temperatures. More generally, the accuracy of any specific electronic structure model can in principle be assessed by comparing the predicted 2D-ACAR spectra with the corresponding observations, although such comparisons to date have been possible only for the local density (LDA) based band theory model, which is the only model where detailed theoretical predictions are currently available.

The early positron studies of $\mathrm{YBa}_{2} \mathrm{Cu}_{3} \mathrm{O}_{7-\mathrm{x}}$ all involved twinned specimens. The first $2 \mathrm{D}$ ACAR results were reported by the Geneva group for projections in the a-b plane [1], and 
from Argonne for the c-projection [2], the latter projection has since been used in most studies due to its suitability in investigating the Fermiology of layered compounds. The initial interpretation of these 2D-ACAR data in terms of the existence of the Fermi surfaces, gave way to a period of lull in the field as subsequent experiments failed to convincingly confirm these conclusions, and thus a consensus concerning a Fermi surface interpretation of the 2D-ACAR data did not emerge in the positron community [3,4]. A comprehensive overview of the work on twinned samples has been given by Barbiellini et al [5].

A new chapter in positron studies of superconductors was opened recently when untwinned $\mathrm{YBa}_{2} \mathrm{Cu}_{3} \mathrm{O}_{7-\mathrm{x}}$ specimens were employed. The first announcement of the untwinned $2 D-A C A R$ results was made by the Texas-Livermore effort [6], followed immediately by the Argonne-Northeastern effort [7]. Most important, the data from these two independent experiments are very similar, and further both groups make a very similar interpretation in terms of the existence of Fermi surfaces in this material. The preliminary results of experiments in Geneva on an untwinned $\mathrm{YBa}_{2} \mathrm{Cu}_{3} \mathrm{O}_{7-x}$ single crystal have recently been reported at the Positron Conference in Hungary (ICPA-9) [8], and appear to be consistent with the aforementioned measurements. It is clear that the coming on line of the untwinned specimens marks a significant milestone, and that the application of the $2 \mathrm{D}$ ACAR technique in exploring the electronic structure and Fermiology of the high- $T_{c}$ 's can now go forward with a degree of confidence.

The crucial importance of model theoretical predictions as a basis for interpreting $2 \mathrm{D}$ ACAR spectra has been evident since the inception of the positron spectroscopy as a tool for investigating the electronic structure and Fermiology of materials. $[9,10]$. The first theoretical electron-positron momentum densities in the high- $T_{\mathcal{c}}$ 's were reported by Bansil et al. [11], and have been followed by a series of theoretical papers by these and other authors [12-16,5]. The basic band theory predictions for the 2D-ACAR spectrum of $\mathrm{YBa}_{2} \mathrm{Cu}_{3} \mathrm{O}_{7}$, and the Fermi surface (FS) signatures therein, have thus been clarified. This theoretical picture has turned out to be robust in that the results from various groups using different detailed band structure methodologies are quite similar, relatively smaller differences in various first principles computations, arising from those in the underlying band structures, notwithstanding. Consequently, these results may be confronted with experiments with a degree of confidence, and have indeed played an essential role in helping interpret 2D-ACAR spectra in $\mathrm{YBa}_{2} \mathrm{Cu}_{3} \mathrm{O}_{7-\mathrm{x}}$.

It is appropriate to make a few brief comments concerning two other commonly used techniques for exploring the electronic structure and Fermiology of materials, i.e. dHvA 
and angle-resolved photoemission (ARPES). Compared to dHvA, the 2D-ACAR experiment possesses the advantage of not requiring long mean free paths, although good quality samples are required due to the sensitivity of the positron to lattice imperfections. Also, since positron annihilation probes the bulk electronic structure, the technique does not suffer from the surface sensitivity of the ARPES. On the other hand, the annihilation process involves all occupied valence electron states, and therefore the amplitude of the spectral features associated with, for example the Fermi surface, is typically only a few percent of the total 2D-ACAR signal. In contrast, $\mathrm{AHvA}$ and ARPES involve selective excitation of a small number of electronic states in the vicinity of the Fermi energy. Bearing these considerations in mind, it makes sense to view dHvA, ARPES, and 2D-ACAR as complementary techniques, and indeed, the most convincing recent evidence for the existence of a Fermi surface in $\mathrm{YBa}_{2} \mathrm{Cu}_{3} \mathrm{O}_{7}$ in essential accord with the band theory predictions, has been pieced together by combining the results of these three spectroscopies.

An outline of this article is as follows. The introductory remarks are followed in Section II by a presentation of experimental details. Notably, the data were collected at temperatures ranging from $30 \mathrm{~K}$ to $300 \mathrm{~K}$, and thus include the superconducting as well as the normal metallic state. Section III delineates the nature of the temperature dependent 2D-ACAR spectra. In Section IV, we present our method of analysis, and the implications of our results for understanding the 2D-ACAR spectra in $\mathrm{YBa}_{2} \mathrm{Cu}_{3} \mathrm{O}_{7}$. We show that the data can be described quite well as a superposition of two temperature independent spectra with temperature dependent relative weights. Most important, we discuss how the temperature dependent data can be used to obtain a 'background corrected' experimental spectrum. As emphasized previously by Bansil et al. [12], a direct comparison of the as observed spectrum with the band theory computations is not sensible because the theoretical spectrum is significantly flatter for low momenta compared to measurements. Section V makes detailed comparisons between such a background corrected 2D-ACAR with the corresponding band theory predictions, followed by a few concluding remarks in Section VI.

\section{Experimental Considerations}

We have obtained 2D-ACAR data in the c-axis projection for untwinned $\mathrm{YBa}_{2} \mathrm{Cu}_{3} \mathrm{O}_{6.9}$ at $30 \mathrm{~K}, 70 \mathrm{~K}, 100 \mathrm{~K}, 185 \mathrm{~K}$ and $300 \mathrm{~K}$. The total number of counts were $100 \mathrm{Mcounts}$ for the $30 \mathrm{~K}$ and $300 \mathrm{~K}$ spectra and $16-22$ Mcounts for the remaining spectra. The resolution (FWHM) due to the camera and the sample size is $0.4-0.5 \mathrm{mrad}$. At low temperatures this 
will be the effective resolution, while the thermal velocity of the positron gives rise to resolution broadening at higher temperatures. Assuming an effective positron mass of one, the overall resolution at $300 \mathrm{~K}$ is $0.7 \mathrm{mrad}$.

The sample was an untwinned single crystal of $\mathrm{YBa}_{2} \mathrm{Cu}_{3} \mathrm{O}_{6.9}\left(0.9 \times 1.6 \times 0.1 \mathrm{~mm}^{3}\right)$ with a transition temperature of $91 \mathrm{~K}$ and a transition width of $1 \mathrm{~K}$. The sample was supported by a $25 \mu \mathrm{m}$ dia. tungsten wire. Based upon separate experiments we estimate the background contribution from the sample support to be $5-10 \%$ of the annihilations. The crystal c-axis was oriented in situ, while the precise a (b) axis orientation was deduced from the data as described below.

For each spectrum the coincidence field and the floodfields used for the efficiency correction were collected intermittently in order to reduce the effects of possibie instrumental instabilities. All data were collected in listmode allowing for a dispersion of 11 $\mu \mathrm{rad} / \mathrm{channel}$ in momentum space. After verifying that the data displayed $\mathrm{C}_{2 \mathrm{v}}$ symmetry, they were subsequently symmerized to augment the signal to noise ratio [17].

The spectral symmetries were studied in a manner similar to that of Ref.[17], i.e. by calculating the Chi-square sum for the hypothesis that the symmetry axis makes a given angle, $\phi$, with the instrumental $p_{x}$ axis. Typical results are shown in Fig. 1 for a twinned as well as an untwinned sample. The minima for the twinned sample are all around 1 , and one concludes that these data possess $\mathrm{C}_{4 \mathrm{v}}$ symmetry. In contrast it is seen that the minima at 45 (135) degrees for the untwinned sample are significantly larger than 1, while the minima at 90 (180) degrees are 1 . Thus the untwinned sample only possesses $\mathrm{C}_{2 \mathrm{v}}$ symmetry. It is also seen from Fig. 1 that the minima do not coincide exactly with 45, 90, 135 and 180 degrees. This is due to a slight misalignment of the sample a (b) axis relative to the instrument axis. By an analysis of Fig. 1 in terms of harmonics one can determine the extent of the misalignment and correct the data accordingly.

\section{Temperature Dependent 2D-ACAR Spectra}

Fig. 2 shows a typical 2D-ACAR spectrum for the untwinned sample, and Fig. 3 gives sections through the temperature dependent $2 \mathrm{D}$-ACAR's along the $\Gamma \mathrm{X}$ and $\Gamma Y$ directions. The temperature dependence is seen to be 'negative' in the sense that the counts at the top of the spectra decrease with temperature. Similar temperature effects have been reported for twinned as well as for polycrystalline samples above the superconducting transition 
temperature [18-20]. Following common practice, the structure in the spectra may be exposed by considering the anisotropy $A(p)$ defined by

$$
A(p)=M(p)-S(p)
$$

where $M(p)$ denotes the measured $2 D-A C A R$ and $S(p)$ is a smooth radially symmetric function which we have chosen to be less than $\mathrm{M}(\mathrm{p})$ for all momenta. The anisotropic distributions, shown in Fig. 4 as a function of temperature, are rather similar in that these consist of a central ridge-like feature surrounded by four mountains. In addition, a less prominent siceridge along the $\Gamma X$ direction is visible at all temperatures at around $12 \mathrm{mrad}$. Notably, the central ridge possesses two peaks whose amplitude decreases with decreasing temperature.

Fig. 5 displays sections through the anisotropic spectra $A(p)$, and provides a more detailed view of these effects. In Fig. 5(a) sections along the $\Gamma X$ direction are shown for various values of py. Fig. 5(b) shows sections perpendicular to ridges seen in Fig. 4. In Fig. 5(b) the ridge is seen as the rapid variation in momentum density near $p_{y}=0$, and the bump around $13 \mathrm{mrad}$ (at the second $\Gamma$ point) arises from the sideridge; for increasing $p_{x}$ values (front to back), the sideridge is seen to gradually overlap with the peaks arising from the 'mountains' centered at the TS direction. In Fig. 5(a), the backmost section shows the two peaks associated with the central ridge; as one moves to higher py values (back to front), one comes down the central ridge and into the peak due to the mountains. Concerning the temperature effects, we see the flattening of the dip around $\left(p_{x}, p_{y}\right)=(0,0)$ in the central ridge (Fig. 5(a), backmost curve) and a somewhat reduced amplitude of the sideridge (Fig. 5 (b)) with decreasing temperature; changes at a finer level in the details of the spectral features as a function of temperature are also apparent.

\section{Treatment of Background}

In comparing band theory predictions with experimental results (for twinned samples), it was noted early that the shapes of the computed and measured 2D-ACAR's differ substantially, the theoretical results being flatter near $\mathrm{p}=0$ in comparison to the measurements; Fig. 6 shows that this discrepancy is present for the twinned specimens as well. In analyzing the twinned data, Bansil et al. [12] found however that if an isotropic 'background' is subtracted from the data, then the theory and experiment agree in many details, leading to the suggestion that the experimental spectra should be corrected for background before making such a comparison. The physical origin of the background is 
not clear, but it arises presumably from defects, oxygen inhomogeneities and other effects not included in the computations which assume a perfect crystal. ${ }^{1}$. Here we reexamine this question and show that a more satisfactory treatment of the background correction may be made in terms of the present temperature dependent spectra.

In order to understand the temperature dependent data, we first examined the possible existence of linear dependencies in the spectra. To accomplish this a new set of spectra,

$$
\mu(p, T)=M(p, T)-\langle M(p)\rangle_{T}
$$

were formed by subracting the temperature averaged spectrum $<\mathrm{M}$ (p) $>\mathrm{T}$ from each of the measured spectra $M(p, T)$. Here all spectra are assumed to be normalized (i.e. $\int \mathrm{M}(\mathrm{p}, \mathrm{T}) \mathrm{d}^{2} \mathrm{p}=1$ ). The $\mu$ spectra (128 by 128 channels each) are organized as five column 'vectors' in a matrix $A_{m, n}\left(m=128^{2}, n=5\right)$. We then pose the question, whether any of the five spectra are linearly dependent on one another. With the question formulated this way, well known matrix techniques can be invoked. Specifically, we carried out the socalled singular value decomposition (SVD) [21] of the matrix A, where each column, as indicated, contains all the data points in one of the 2D-ACAR spectra. Obviously there can at most be four linearly independent $\mu$ spectra. The SVD analysis yielded four orthonormal basis vectors (denoted $U^{i}(p)$ for $i=1$ to 4 ) and each physical spectrum, $M(p, T)$, can then be

${ }^{1}$ The calculations of Ref. $[11,12]$ do not include electron-positron enhancement of the momentum density; the inclusion of the enhancement effect makes the theoretical $2 \mathrm{D}-\mathrm{ACAR}$ more peaked near $p=0[5,14,16]$, but the essential discrepancy between the theory and experiment seems to remain. It is also possible that a more sophisticated theory of the highly correlated electron gas (beyond the LDA) may naturally lead to a better shape of the 2D-ACAR near $p=0$. It is important to recognize however that all such electron-electron and electron-positron correlation effects would normally be expected to be temperature independent, and would thus not explain the observed temperature dependence of the 2D-ACAR. For this reason, it seems reasonable to invoke some sort of a background correction when comparing theory with experiment. It is of course possible that the observed discrepancy is in part due to correlation effects not being taken properly into account, and in part due to a temperature dependent background effect. 
written as a linear combination of the $\mathrm{U}^{\mathrm{i}}(\mathrm{p})$ 's with associated temperature dependent coefficients $\alpha_{i}(T)$.

$$
M(p, T)=\langle M(p)\rangle_{T^{+}}+\sum_{i=1}^{4} \alpha_{i}(T) L^{-i}(p)
$$

Inspection showed that one of the coefficients dominated with a 5 times larger temperature dependence than the remaining coefficients. We thus arrive at the approximate representation:

$$
\mathrm{M}(\mathrm{p}, \mathrm{T}) \approx\langle\mathrm{M}(\mathrm{p})\rangle_{\mathrm{T}}+\alpha_{1}(\mathrm{~T}) \mathrm{L}^{-1}(\mathrm{p})
$$

where $\alpha_{1}(\mathrm{~T})$ denotes the coefficient with the largest variation. The most important implication of equation 4 is that the data can be expressed to a good approximation as a weighted average of two temperature independent spectra.

Eq. 4 also implies that $U^{1}(p)$ is essentially proportional to the difference between the spectra at any two temperatures, a typical result is shown in Fig. 7. The dark square-like region in the center of Fig. 7(b) nearly coincides with the first Brillouin zone, indicating that the overall effect with decreasing temperature consists of an increase in the momentum density at the center of the zone (i.e. a spectrum more peaked at the center). There is a concomitant decrease in the momentum density in primarily the second zone; this effect is seen from Figs. 7(a) and 7(b) to be quite anisotropic, the negative region around $5 \mathrm{mrad}$ along $\Gamma X$ being deeper than along the $\Gamma Y$ direction. This reduction in the umklapp probability is unusual because in most metals one observes a corresponding increase with decreasing temperature (i.e. a distribution which becomes less peaked at the center). It is interesting in this connection to consider the effects of shallow traps, i.e. regions of the lattice with positron binding energies of order $\leq 10 \mathrm{meV}(100 \mathrm{~K})$; these should be contrasted to the more usual vacancy type traps with binding energies on the order of $1 \mathrm{eV}$. At low temperatures some of the positrons may then annihilate in the trapped state; at higher temperatures the positron may detrap prior to annihilation due to thermal excitations, and most of the annihilations will then take place while in the delocalized Bloch-like state (e.g.[22]). Since one generally expects the annihilation spectrum for a trapped positron state (which is localized) to be narrower than for a delocalized Bloch-like state, it is then clear that shallow traps are a possible mechanism for explaining some of the observed 
temperature dependence of the 2D-ACAR in $\mathrm{YB}_{2} \mathrm{Cu}_{3} \mathrm{O}_{7-\mathrm{x}}$. The effects of various traps are considered in further detail in Ref.[23].

We return now to equation (4) which may be cast in the equivalent form:

$$
\begin{aligned}
\mathrm{M}(\mathbf{p}, \mathrm{T}) & =\mathrm{F}(\mathbf{p})+\mathrm{I}(\mathrm{T})(\mathrm{B}(\mathrm{p})-\mathrm{F}(\mathrm{p})) \\
& =(1-\mathrm{I}(\mathrm{T})) \mathrm{F}(\mathrm{p})+\mathrm{I}(\mathrm{T}) \mathrm{B}(\mathrm{p})
\end{aligned}
$$

where we have formally replaced the spectra $\langle\mathrm{M}(\mathrm{p})\rangle_{\mathrm{T}}$ and $\mathrm{U}^{1}(\mathrm{p})$ occurring in equation (4) by two other spectra $F(p)$ and $B(p)$. The motivation for so recasting equation (4) is that form 5 (b) is often invoked in positron defect spectroscopy in connection with annihilation spectrum from a sample with a singie species of defects, where $F(p)$ and $B(p)$ correspond to the spectra from the perfect bulk material and the defects respectively, and $I(T)$ to the trapping probability. The fact that the present spectra admit a representation of form (5) implies that our temperature dependent data may be viewed as a superposition of two types of annihilations. It makes sense to consider one of these as the bulk annihilations, $F(p)$, and the remainder as 'background' annihilations, $B(p)$. We emphasize that the physical origin of such a background is unclear, although, keeping the discussion of Fig. 7 above in mind, it is reasonable to interpret the background to be the result of shallow traps.

In defect spectroscopy (see e.g.[24]), the spectra corresponding to $F(p)$ and $B(p)$ can be determined from direct observations in the limiting situations, i.e, at high temperatures there may be no trapping so that $\mathrm{I}(\mathrm{T})=0$, and at low temperatures there may be complete trapping which implies $I(T)=1$. There is no indication that in the present case any of the measured spectra represent such limiting cases, leaving the forms of the spectra $F(p)$ and $B(p)$ experimentally undetermined. Despite this, we show below that progress is possible in assessing the extent to which the experimental results are consistent with the theoretically predicted 2D-ACAR spectrum, $T(p)$, for bulk annihilations.

To this end, note that by writing an equation of form $5(\mathrm{~b})$ at two different temperatures $T_{1}$ and $T_{2}$, we may formally eliminate the term $B(p)$, and thus express $F(p)$ as a linear combination of any two measured spectra $M\left(p, T_{1}\right)$ and $M\left(p, T_{2}\right)$, i.e.

$$
F(p)=(1-\beta) M\left(p, T_{1}\right)+\beta M\left(p, T_{2}\right)
$$

where $\beta$ is easily obtained in terms of $I\left(T_{1}\right)$ and $I\left(T_{2}\right)$. We now argue that we may make an absolute comparison between theory and experiment by fitting the constant $\beta$ on the right 
side of (6) to the resolution-broadened band theory predicted spectrum $T(p)$ so that we can write ${ }^{2}$ :

$$
T(p) \approx(1-\beta) M\left(p, T_{1}\right)+\beta M\left(p, T_{2}\right)
$$

Denoting the fitted value by $\widetilde{\beta}$, we thus obtain the distribution

$$
H(p) \equiv(1-\tilde{\beta}) M\left(p, T_{1}\right)+\tilde{\beta} M\left(p, T_{2}\right)
$$

where $\widetilde{\beta}$ is the important factor which determines the shape of the spectrum. $H(p)$ can be considered a 'background corrected' experimental spectrum. The specific value of $\tilde{\beta}$ was determined by using only the $\Gamma Y$ theoretical and experimental sections (if another direction is used in the fitting procedure the results are similar). We obtain however a good fit between theory and experiment not only along $\Gamma Y$ but in many other directions as well. In order to obtain the best statistical accuracy, the $30 \mathrm{~K}$ and $300 \mathrm{~K}$ pair of spectra were used to obtain the constant $\widetilde{\beta}^{3}$ ).

Finally, we emphasize that while the preceding procedure determines the bulk spectrum $F(p)$ from the experimental spectra in terms of a single parameter $\beta$, the background spectrum $B(p)$ is not determined; information obtained independently of the present data will be needed to determine $B(p)$ uniquely. We have however made some estimates of the magnitude of the total background contribution to the $300 \mathrm{~K}$ spectrum. The maximum value can be shown to arise under the assumption that the background is given by the $30 \mathrm{~K}$ spectrum, which yields an estimate of $70 \%$ background in the $300 \mathrm{~K}$ data. On the other hand if we determine the background under the constraint that it be as small as possible (and be positive definite), we obtain a value of $15-20 \%$ as an estimate of the lower bound for the background in the $300 \mathrm{~K}$ spectrum.

\footnotetext{
${ }^{2}$ Formally, the overall normalization factor between theory and experiment is also an adjustable parameter, factorizing the right hand side of eq(7). Although this factor can be calculated from theory, this value cannot be used in the present context, since the experimental data cannot be normalized in an infinite momentum region; this is especially true because of the small unknown contributions from small-angle scattering in the sample and instrument collimators.

${ }^{3}$ It should be noted that the fitting procedure results in a spectrum with a noticeable higher statistical error than the as observed spectra. This has been taken into account in what follows.
} 


\section{Background Corrected Data and Band Theory Predictions}

Fig. 8 compares the background corrected spectrum, $\mathrm{H}(\mathrm{p})$, with the normalized theoretical spectrum, $T(p)$, along the high symmetry directions in the first as well as higher Brillouin zones. A reference to Figs. 6 and 8 shows a dramatic improvement with regard to the overall agreement between the theory and experiment. Notably, the as observed data (Fig. 6 ) is rather similar along the $\Gamma X$ and $\Gamma Y$ directions, while the corrected data (Fig. 8) exhibits clear anisotropy near $\mathrm{p}=0$ in good accord with the band theory predictions.

Turning to more detailed features of the spectra, the anisotropy in the form of the difference section $\Gamma Y-\Gamma X$ (i.e. the difference between the sections along the $\Gamma Y$ and $\Gamma X$ directions) for the $30 \mathrm{~K}, 300 \mathrm{~K}$, and the background corrected $(\mathrm{H}(\mathrm{p}))$ data is compared in Fig. 9 with the band theory predictions. The shapes of the three experimental curves in Fig. 9 are similar in the sense that they all possess a negative lobe followed by a less prominent positive lobe, and display a steep decrease near zero momentum. The positive lobe in the $30 \mathrm{~K}$ data is however smaller than the other data sets, and the shoulder around $6.3 \mathrm{mrad}$ is seen in both the $300 \mathrm{~K}$ and the background corrected data is hardly visible at $30 \mathrm{~K}$. Most important, the amplitude of the anisotropy for the $30 \mathrm{~K}$ as well as the $300 \mathrm{~K}$ spectrum is about a factor of two too small compared to theory; in contrast, the background corrected data agrees well with the theoretical predictions in this regard for the first time. We emphasize that it is not satisfactory to obtain such an agreement by simply scaling the $30 \mathrm{~K}$ or the $300 \mathrm{~K}$ results of Fig. 9 since all data sets in the figure possess the same normalization. We note finally that the results for the absolute anisotropy discussed in this paragraph with reference to the $\Gamma Y-\Gamma X$ difference are typical of the other difference sections such as $\Gamma \mathrm{X}-\Gamma \mathrm{S}, \Gamma \mathrm{Y}-\Gamma \mathrm{S}$, and XS-YS (not shown), which we have considered.

We discuss now the FS signatures in the background corrected data $\mathrm{H}(\mathbf{p})$. In what follows we will frequently refer to the four FS-sheets $[25,26]$ predicted by the LDA, namely: (1) a pair of closely spaced S-centered hole sheets, which possess the shape of the inner and outer surfaces of a 'barrel', (2) an electron 'ridge' surface running along $\Gamma X$, and (3) a 'pillbox' hole sheet centered at $S$ along with an associated hole sheet mainly centered on the ГY direction. Most calculations agree well with regard to the 'barrels' and the 'ridge'. Some relatively minor differences are however found with regard to the 'pillbox' and the associated hole sheets; this is expected since these sheets arise from a heavy mass band that lies close to the Fermi level and are therefore sensitive to computational details. 
In order to enhance FS signatures, anisotropies in the form of difference sections for several pairs of directions are considered in Fig. 10 for the background corrected data. We comment on the theoretical results of Fig. 10(a) first. The complexity of these spectra arises not only from the presence of various FS sheets in $\mathrm{YBa}_{2} \mathrm{Cu}_{3} \mathrm{O}_{7}$, but also from the detailed character of the electronic wave functions of various filled bands. Several of the 'breaks' associated with the Fermi surface are indicated (e.g, $\mathrm{r}_{1}, \mathrm{r}_{2}, \mathrm{~s}_{1}, \ldots$ etc.). The sharpness of such breaks reflects the nearly dispersionless nature of the band structure of $\mathrm{YBa}_{2} \mathrm{Cu}_{3} \mathrm{O}_{7}$ along the $\mathrm{c}$-axis, and emphasizes the advantage of using the $\mathrm{c}$-axis projection in exploring the Fermiology of lavered materials. Note that the FS breaks in Fig. 10(a) occur in a distribution which itself possesses substantial underlying structure.

The signatures of various FS sheets in Fig. 10(a) are as follows. The ridge FS gives the rapid variation $\left(\mathrm{r}_{0}\right)$ near $\mathrm{p}=0$; since the ridge lies along the $\Gamma X$ direction, in the $\Gamma S-\Gamma X$ spectrum or other difference spectra involving the $\Gamma$-point, the anisotropy is nearly zero for momenta (around $\mathrm{p}=0$ ) of the order of the width of the ridge. The first umklapp of the ridge $\left(\mathrm{r}_{1}, \mathrm{r}_{2}\right)$ is seen clearly around $6.3 \mathrm{mrad}$ as a peak in $\Gamma Y-\Gamma X$, and a dip in $\Gamma S-\Gamma Y$; only weak features are seen at this momentum in the $\Gamma S-\Gamma X$ or XS-YS curves. The second image of the ridge $\left(r_{3}, r_{4}\right)$ occurs around 13 mrad. The high momentum end $r_{4}$ is generally more clearly visible than the low momentum feature $r_{3} ; r_{4}$ in particular coincides with the zero crossing in $\Gamma S-\Gamma Y$. The pillbox FS yields relatively prominent features only in the XS-YS spectrum. The breaks $s_{1}, s_{2}, s_{3}$, and $s_{4}$ are all associated with the pillbox. Since the dimensions of the pillbox in the XS and YS directions are different, but the XS-YS difference must obviously vanish at the S-point (around $3.1 \mathrm{mrad}$ ), we get two breaks on each side of the S-point in the XS-YS spectrum. Interestingly though the image of the pillbox around $9.5 \mathrm{mrad}\left(\mathrm{s}_{5}, \mathrm{~s}_{6}\right)$ only consists of two prominent breaks. This results from the fact that in this momentum region, the pillbox possesses a substantial signature only along the YS and not the XS direction. Finally, as expected the $\mathrm{Cu}-\mathrm{O}$ plane sheets give essentially no discernible signatures in the 2D-ACAR's as a result of the weak coupling of the positron Bloch state with the $\mathrm{Cu}-\mathrm{O}$ planes [11-16].

Fig. 10(b) shows that experimental anisotropies are in good accord with theory with regard to the details of the spectral features, the agreement concerning the absolute magnitude of the overall undulations have already been noted above. The level of discrepancies seen here is common in even simpler materials in first principles comparisons between theory and experiment. Notably, in a twinned crystal, the XS-YS and $\Gamma Y-\Gamma X$ anisotropy is zero, and 
$\Gamma S-\Gamma X$ is identical to $\Gamma S-\Gamma Y$. Therefore important aspects of the momentum density simply cannot be probed via measurements on twinned crystals.

FS signatures in the experimental spectra can now be elucidated with reference to Fig. 10(b). We consider the ridge FS first. As noted above, the ridge FS gives three distinct features: (1) rapid variation in anisotropy near $p=0,(2)$ an image around $6.3 \mathrm{mrad}$, and (3) a second image around $13 \mathrm{mrad}$. The experimental spectra display all three of these features. Although we do not of course expect any 'breaks' in the experimental data due to resolution effects, near $\mathrm{p}=0$, both sets of curves in Fig. 10(b) possess similarly rapid variations and slopes: the width of the experimental ridge would however appear to be somewhat larger than that implicit in our band structure. The $6.3 \mathrm{mrad}$ image of the ridge $\left(\mathrm{r}_{1}, \mathrm{r}_{2}\right)$ is particularly important in the $\Gamma Y-\Gamma \mathrm{X}$ curve because this feature lies on a relatively gently varying background and remains quite prominent in the theory even after including resolution broadening, and it is crucial that this feature is indeed observed clearly in the experimental data. By contrast, in the $\Gamma S-\Gamma Y$ curve, the resolution broadening effects would make the $6.3 \mathrm{mrad}$ ridge image difficuit to distinguish from the background. Finally, the image of the ridge $F S$ near 13 mrad $\left(r_{3}, r_{4}\right)$ becomes rather smooth in theory upon resolution broadening. We emphasize however that the experimental data in this momentum range is quite consistent with the theory, and in particular, Fig. 11 which gives a blow up of this region, shows that the experimental edge around $13 \mathrm{mrad}$ is just about as sharp as that predicted theoretically. Also, in the case of $\Gamma S-\Gamma Y$, both theory and experiment show the related zero crossing around $13 \mathrm{mrad}$.

Another interesting observation concerning the $13 \mathrm{mrad}$ FS feature may be made with reference to Fig. 11. The right edge in this figure around $13 \mathrm{mrad}$ is roughly as sharp as the left edge around $8.5 \mathrm{mrad}$, even though the latter is not interpreted to be a FS feature. In this sense, the $13 \mathrm{mrad}$ ridge feature is intinsically a 'weak' signature (compared to the features around $p=0$ and $6.3 \mathrm{mrad}$ ) and by examining the experimental data of Fig. 11 alone, one would be hard pressed to convincingly associate the $13 \mathrm{mrad}$ edge with an FS. It turns out however that as one considers other parallel sections through the 2D-ACAR distribution perpendicular to the ridge [6,7], the left edge quite rapidly changes shape, while the $13 \mathrm{mrad}$ feature yields a rather pronounced flat feature and thus provides a reasonable evidence for its being of an FS origin.

Concerning the pillbox, as noted, the prominent features involve the XS-YS anisotropic spectrum, where the theory and experiment appear to be in accord in Fig. 10(b), although upon resolution broacening, the theoretical breaks $\mathrm{s}_{1}$ through $\mathrm{s}_{4}$ become rather indistinct, 
and are difficult to identify in the experimental spectra. The dip arising from the breaks $\mathrm{s}_{5}$ and $s_{6}$ remains distinct even after resolution effects are included, and in this momentum region, the experimental data display a similar dip in accord with the theory. Further analysis of the $2 \mathrm{D}-\mathrm{ACAR}$ data is nevertheless needed to firmly establish the existence of the pillbox FS via the present experiments. Finally, it may be noted that the present results are complementary to the $\mathrm{dHvA}$ and angle resolved photoemission results concerning the Fermi surface features [27-29].

\section{Conclusions.}

In summary, we have carried out $2 \mathrm{D}-\mathrm{ACAR}$ positron experiments on an untwinned single crystal of metallic $\mathrm{YBa}_{2} \mathrm{Cu}_{3} \mathrm{O}_{7-\mathrm{x}}$ as a function of temperature over the range $30 \mathrm{~K}$ to $300 \mathrm{~K}$. We show that, to a good approximation, the temperature dependent data can be described as a superposition of two temperature independent spectra whose relative weights vary with temperature. On this basis, we suggest that the observed spectra may be viewed as resulting mainly from two mechanisms, namely, annihilations involving the positron in the Bloch-state, and other 'background' annihilations. The detailed nature of the 'background' annihilations is unclear, although the temperature dependence suggests that the process would involve one (or more) shallow traps. We discuss in detail how the temperature dependent data can be used to extract a 'background corrected' experimental spectrum, and compare the results so obtained with the corresponding band theory predictions by considering many sections through the two-dimensional distribution. The background corrected data is in remarkable agreement with the band theory predictions, including for the first time the overall amplitude of the anisotropies. The earlier analysis of the twinned as well as the untwinned data has invoked an isotropic background (of weight determined by making the best fit between theory and experiment), but yielded a puzzling discrepancy in this regard so that the experimental anisotropies were about a factor two too small compared to the band theory; the present treatment implies background with sufficient anisotropy that this discrepancy is essentially removed. The corrected data also shows clear signatures of the ridge Fermi surface and an indication of the pillbox surface.

\section{Acknowledgments:}

We gratefully acknowledge discussions with Dr. R.Benedek and Dr. P.E. Mijnarends. The present work was supported by the U.S. Department of Energy, Basic Energy Sciences, Division of Materials Sciences under contract \#W-31-109- ENG-38 (LS, YF, KB), including a subcontract to Northeastern University $(A B)$, and the National Science 
Foundation's Office of Science and Technology Centers under contract \#STC-8809854 (UW). This work benefited from the allocation of supercomputer time on the NERSC and San Diego Supercomputer centers. 


\section{References}

[1] L. Hoffmann, A.A. Manuel, M. Peter, E. Walker, and M.A. Damento Europhys Lett. 661 (1988).

L. Hoffmann, A.A. Manuel, M. Peter, E. Walker, and M.A. Damento Physica C 153-155 129

(1988).

M. Peter IBM J. Res. Dev. 33333 (1988).

[2] L.C. Smedskjaer, J Z. Liu, R. Benedek, D.G. Legnini, D.J. Lam, M.D. Stahulak, H. Claus , and A. Bansil Physica C 156269 (1988).

[3] M. Peter and A.A. Manuel Phys. Scr. T29 106 (1989).

[4] H. Haghighi, J.H. Kaiser, S. Rayner, R.N. West, M.J. Fluss, R.H. Howell, P. E. A. Turchi, A.L. Wachs, Y.C. Jean, and Z.Z. Wang J. Phys. Condens. Matter $2 \underline{2} 1911$ (1990)

[5] B. Barbiellini, P.Genoud, J.Y. Henry, L.Hoffmann, T. Jarlborg, A.A. Manuel, S. Massida, M.Peter, W. Sadowski, H.J. Scheel, A. Shukla, A.K. Singh, and E. Walker Phys Rev B $43(10)$ 7810 (1991).

[6] H. Haghighi, J.H. Kaiser, S. Rayner, R.N. West, J.Z. Liu, R. Shelton, R.H. Howell, F. Solar, and M.J. Fluss: Phys. Rev. Lett. 67, 382 (1991); J. Phys. Chem. Solids 52, 1535 (1991)

[7] L. C. Smedskjaer, A. Bansil, U. Welp, Y. Fang, and K. G. Baily, J. Phys. Chem. Solids 52 , 1541 (1991)

[8] L. Hoffmann, W. Sadowski, and M. Peter, Proceedings of the 9'th "Int. Conf. on Positron Annihilation", Hungary (1991)

[9] S. Berko in, Momentum Distributions, R. N. Silver and P. E. Sokol, eds. (Plenum, New York, 1989), p273

[10] P. E. Mijnarends, in Positrons in Solids, Course LXXXIII, International School of Physics, "Enrico Fermi" (North-Holland, Amsterdam, 1982)

[11] A. Bansil, R. Pankaluoto, R.S. Rao, P.E. Mijnarends, W. Dlugosz, R. Prasad, and L.C. Smedskjaer, Phys. Rev. Lett. 61, 2480 (1988).

[12] A. Bansil, P. E. Mijnarends, and L. C. Smedskjaer, Physica C172, 175(1990)

A. Bansil, J. Phys. Chem. Solids 52 , 1493 (1991)

[13] A. Bansil, P. E. Mijnarends, and L. C. Smedskjaer, Phys. Rev. B43, 3667(1991)

[14] S. Massidda, Physica C169, 137(1990)

[15] D. Singh, W. E. Pickett, E. C. von Stetten, and S. Berko, Phys. Rev. B42, 2696(1990)

[16] T. Jarlborg, B. Barbiellini, E. Boronski, P. Genoud, and M.Peter, J. Phys. Chem. Solids 52, 1515 (1991)

[17] L.C. Smedskjaer and D.G. Legnini, Nucl. Inst. Meth. Phys. Res. A292, 487 (1990)

[18] L. Hoffmann, W. Sadowski, A. Shukla, Gh. Adam, B. Barbiellini, and M. Peter, J. Phys. Chem. Solids 52,1551 (1991)

[19] L.C. Smedskjaer, B.W. Veal, D.G. Legnini, A.P. Paulikas, and L.J. Nowicki Phys. Rev. B 37 2330 (1988); Physica B 150, 56 (1988)

[20] E.C. von Stetten, S. Berko, X.S. Li, R.R. Lee, J. Brynestad, D. Singh, H. Krakauer, W.E. Pickett, and R.E. Cohen, Phys. Rev. Lett. 60, 2198 (1988)

[21] G.H. Golub, and C.F. Van Loan, Matrix Computations 1983, Johns Hopkins University Press (Baltimore), Chapter 12.

[22] L.C. Smedskjaer, M. Manninen, and M.J. Fluss, J. Phys. F., 10 ,2237, (1980)

[23] K.O. Jensen, R.M. Nieminen, and M.J. Puska, J. Phys.: Condensed Matter 1, 3727 (1989)

[24] M.J. Fluss, S. Berko, B. Chakraborty, K.R. Hoffmann, P. Lippel, and R.W. Siegel, J. Phys. F: Met. Phys, 14, 2831 (1984)

[25] J.Yu, S.Massidda, A.J.Freeman, and D.D.Koelling, Phys. Lett. A122, 203 (1987)

[26] W. E. Pickett, R. E. Cohen, and H. Krakauer, Phys. Rev, B42, 8764(1990)

[27] J. C. Campuzano, L. C. Smedskjaer, R. Benedek, G. Jennings, and A. Bansil, Phys. Rev. B43, 2788(1991)

[28] F. M. Mueller, J. Phys. Chem. Solids 52,1457 (1991), J.L. Smith, C. M. Fowler, B. L. Freeman, W. L. Hults, J. C. King, and F. M. Mueller to appear in Proc. of third Int. Symp. on Superconductivity, Sendai Japan.

[29] G. Kido, K. Komorita, H. Katayama-Yoshida, and T. Takahashi, J. Phys. Chem. Solids 52, 1465 (1991) 


\section{Figure Captions}

Fig. 1. $\chi^{2}$ sum vs. angle of hypothetical symmetry axis: untwinned sample at $300 \mathrm{~K}$ (curve a), untwinned sample at $30 \mathrm{~K}$ (curve b), and twinned sample at $30 \mathrm{~K}$ (curve c). Solid curves are harmonic fits to the data.

Fig. 2 A typical as observed 2D-ACAR spectrum for untwinned $\mathrm{YBa}_{2} \mathrm{Cu}_{3} \mathrm{O}_{6.9}$; resuits are here shown for the $30 \mathrm{~K}$ measurement.

Fig. 3 Sections along the $\Gamma X$ and $\Gamma Y$ directions through the as observed $2 \mathrm{D}-\mathrm{ACAR}$ spectra at $30 \mathrm{~K}, 185 \mathrm{~K}$, and $300 \mathrm{~K}$ (top to bottom). The data for $70 \mathrm{~K}$ and $100 \mathrm{~K}$ (not shown) lie between the $30 \mathrm{~K}$ and $185 \mathrm{~K}$ curves.

Fig. 4 A three-dimensional rendition of the anisotropic spectra $A(p)$ (see Eq. (1)) from untwinned $\mathrm{YBa}_{2} \mathrm{Cu}_{3} \mathrm{O}_{6.9}$ at various temperatures. The pixel size is $1.57 \mathrm{mrad}$ by $1.57 \mathrm{mrad}$.

Fig. 5. Sections through the anisotropic distributions A(p) of Fig. 4. A channel size of $0.39 \mathrm{mrad}$ is used here. (a): Cuts along $\Gamma X$, i.e. the horizontal axis is the momentum $p_{x}$. Note that in order to display the structure in the data, $p_{y}$ is chosen to decrease from front to back, so that in each frame the front curve is for $p_{y}=\pi / b$, while the backmost curve is for $p_{y}=0$. The first long tickmark on the $\mathrm{p}_{\mathrm{x}}$-axis (inside the frame) denotes the $\mathrm{Y}$ point, and the others the images of $\mathrm{Y}$ at higher momenta. (b): Cuts along $\Gamma \mathrm{Y}$, much like in (a) above. Note however that here the structure is better displayed by choosing $p_{x}$ to increase from front to back, so that the front curve is for $p_{x}=0$ and the backmost curve for $p_{x}=\pi / a$. The first long tickmark on the $p_{y}$-axis (inside the frame) denotes the $\Gamma$ point, while the others give images of $\Gamma$ at higher momenta. The relative offsets between the curves are $1 \mathrm{mrad}$ in the horizontal and $1 \cdot 10^{-5} \mathrm{mrad}^{-2}$ in the vertical directions respectively.

Fig.6 Comparison between theory and the as observed sections through typical 2DACAR spectra for untwinned (a) and twinned (b) $\mathrm{YBa}_{2} \mathrm{Cu}_{3} \mathrm{O}_{7-\mathrm{x}}$ samples. The results are shown for the $300 \mathrm{~K}$ measurements.

Fig. 7 Difference between normalized $30 \mathrm{~K}$ and $300 \mathrm{~K}$ 2D-ACAR spectra for untwinned $\mathrm{YBa}_{2} \mathrm{Cu}_{3} \mathrm{O}_{6.9}$, shown as, (a): a three dimensional rendition, and (b): 
as a contour plot. The $30 \mathrm{~K}$ spectrum is higher than the $300 \mathrm{~K}$ spectrum near $\mathrm{p}=0$. The boundaries for the first zone are shown.

Fig. 8 A comparison of various sections through the background corrected 2D-ACAR spectrum, $\mathrm{H}(\mathrm{p})$, for the untwinned $\mathrm{YBa}_{2} \mathrm{Cu}_{3} \mathrm{O}_{6.9}$ sample with the corresponding theoretical predictions of Bansil et al. [12]. The results for the high symmetry lines $\Gamma Y, \Gamma X, \Gamma S$, YS, and XS and parallel lines through higher zones are shown as marked.

Fig. 9 A comparison between the $\Gamma \mathrm{Y}-\Gamma \mathrm{X}$ anisotropy for the normalized $30 \mathrm{~K}, 300 \mathrm{~K}$, and the background corrected data $\mathrm{H}(\mathrm{p})$, with the corresponding resolution broadened band theory predictions of Ref. [12]. The spectra have been offset relative to one another as indicated by the horizontal lines.

Fig. 10 (a): Theoretical anisotropic momentum distribution, here defined by taking differences between spectra along four different pairs of directions. Thin vertical lines mark the position of various $F S$ features $r_{0}, s_{1}$, etc. discussed in the text. (b) A comparison between the resolution broadened theory curves in (a) with the corresponding experimental difference sections obtained from the corrected data $\mathrm{H}(\mathbf{p})$.The spectra have been offset relative to one another as indicated by the horizontal lines.

Fig. 11 A blow up of the $8 \mathrm{mrad}$ to $16 \mathrm{mrad}$ momentum region of the $\Gamma Y-\Gamma X$ anisotropic sections of Fig. 10(b). In order to emphasize shapes the data has been scaled to produce a visual agreement between the amplitudes of the theoretical and experimental curves. Experimental data have been averaged over $1.57 \mathrm{mrad}$ in the $\Gamma X$ direction. 


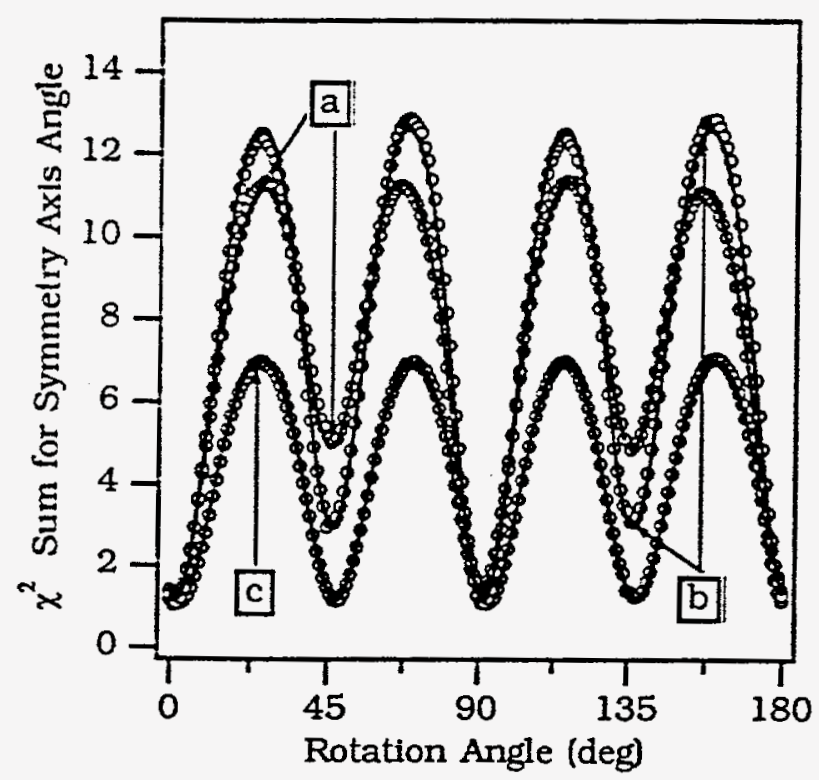

Fig. 1 Smedskjaer et al 1992 


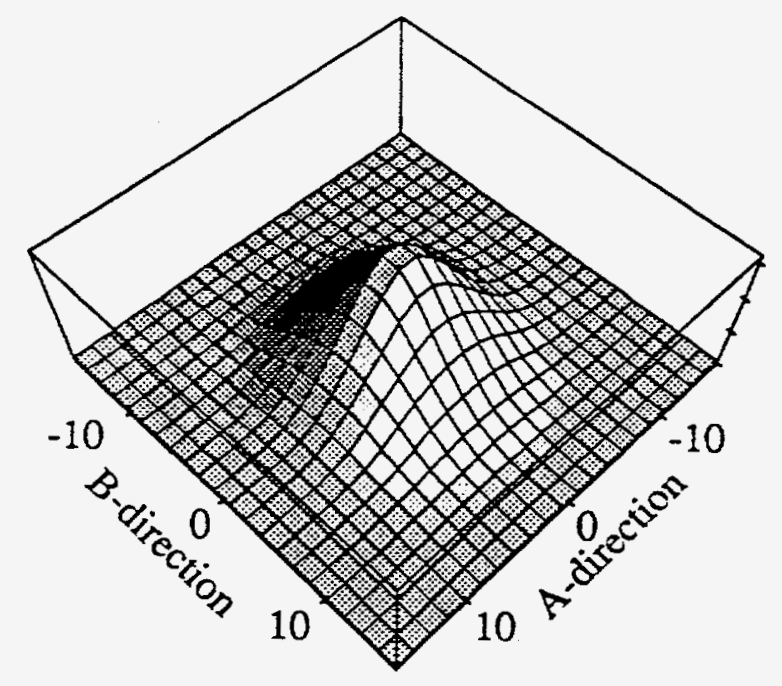

Fig. 2 Smedskjaer et al 1992 


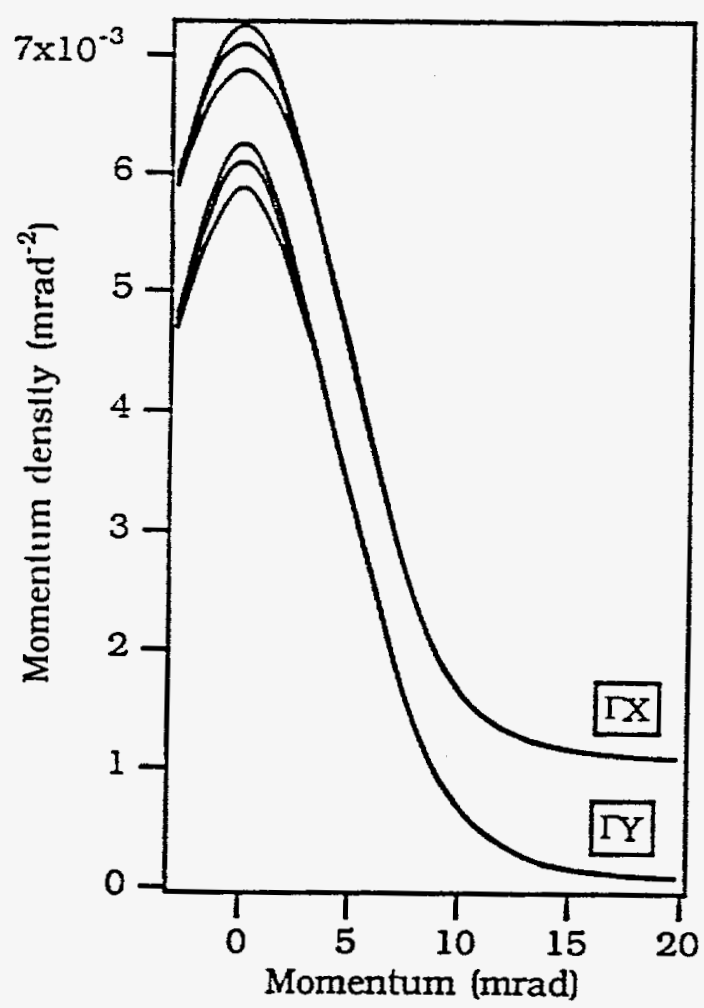

Fig 3 Smedskjaer et al 1992 


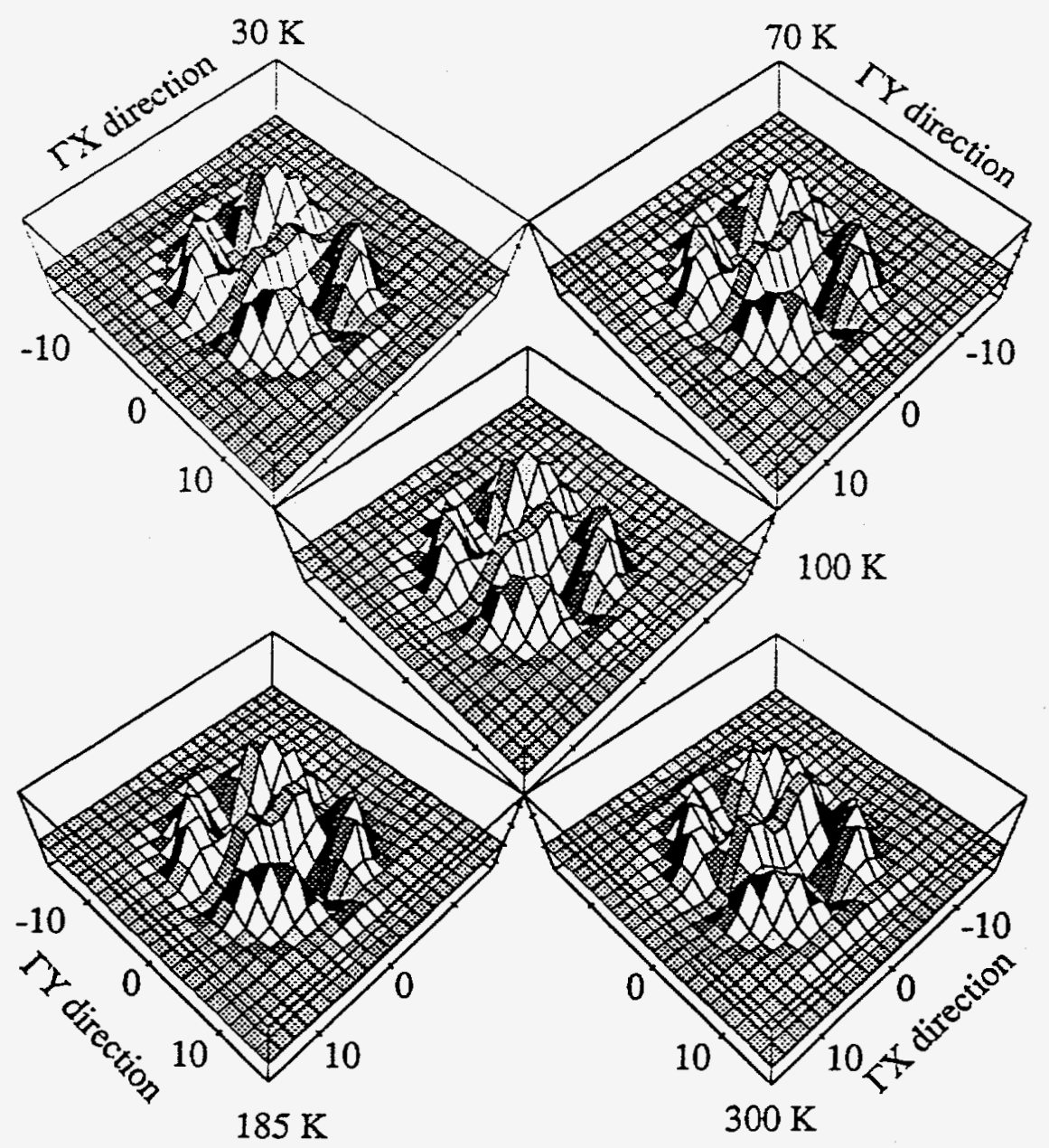

Fig. 4 Smedskjaer et al 1992 
(a)

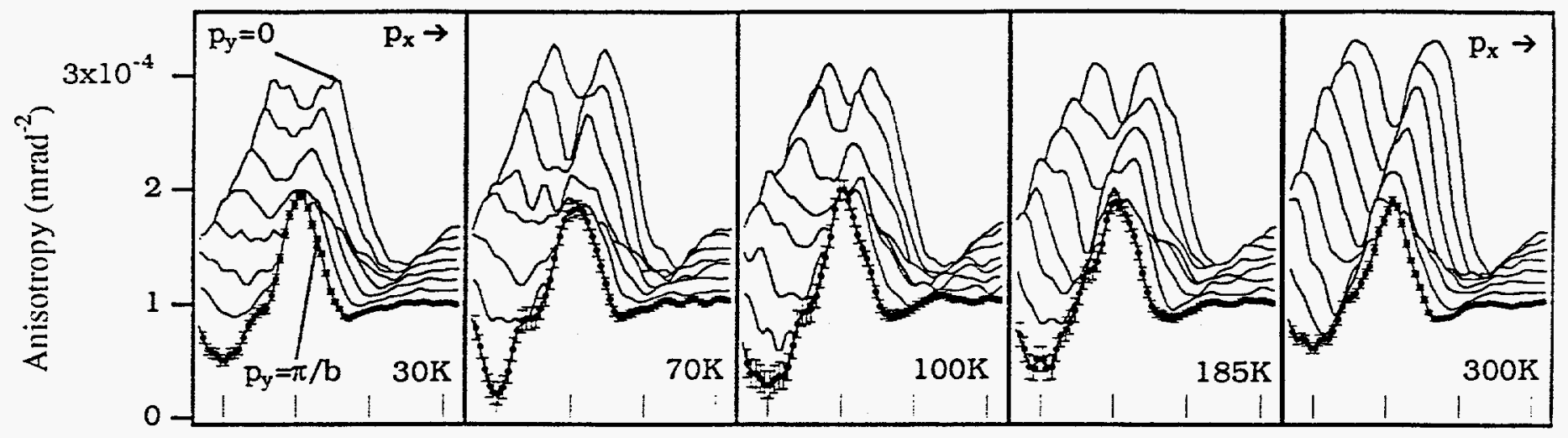

(b)

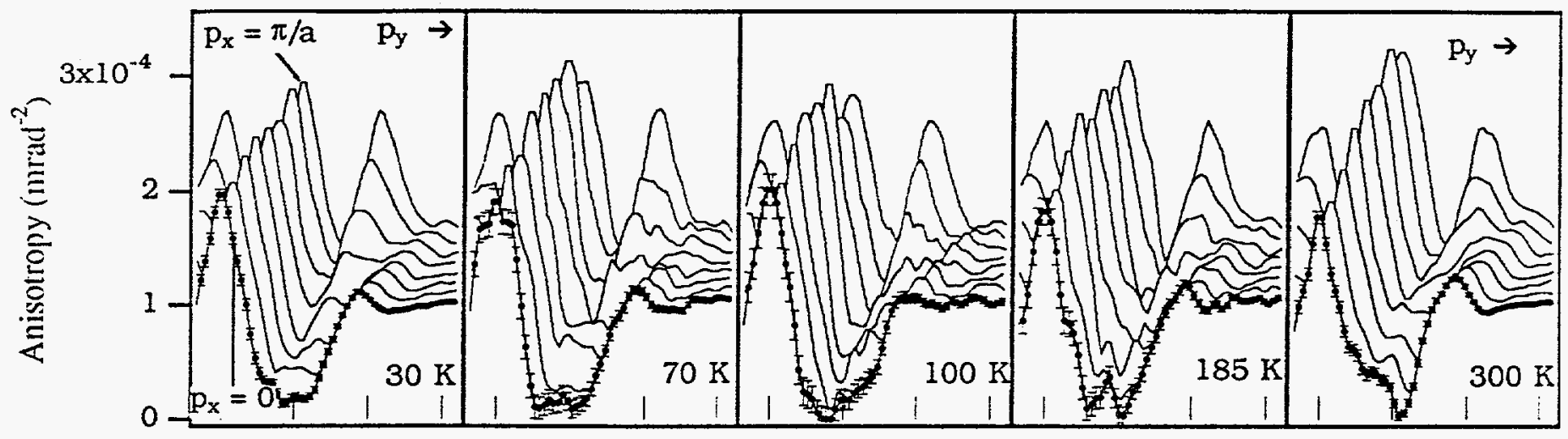



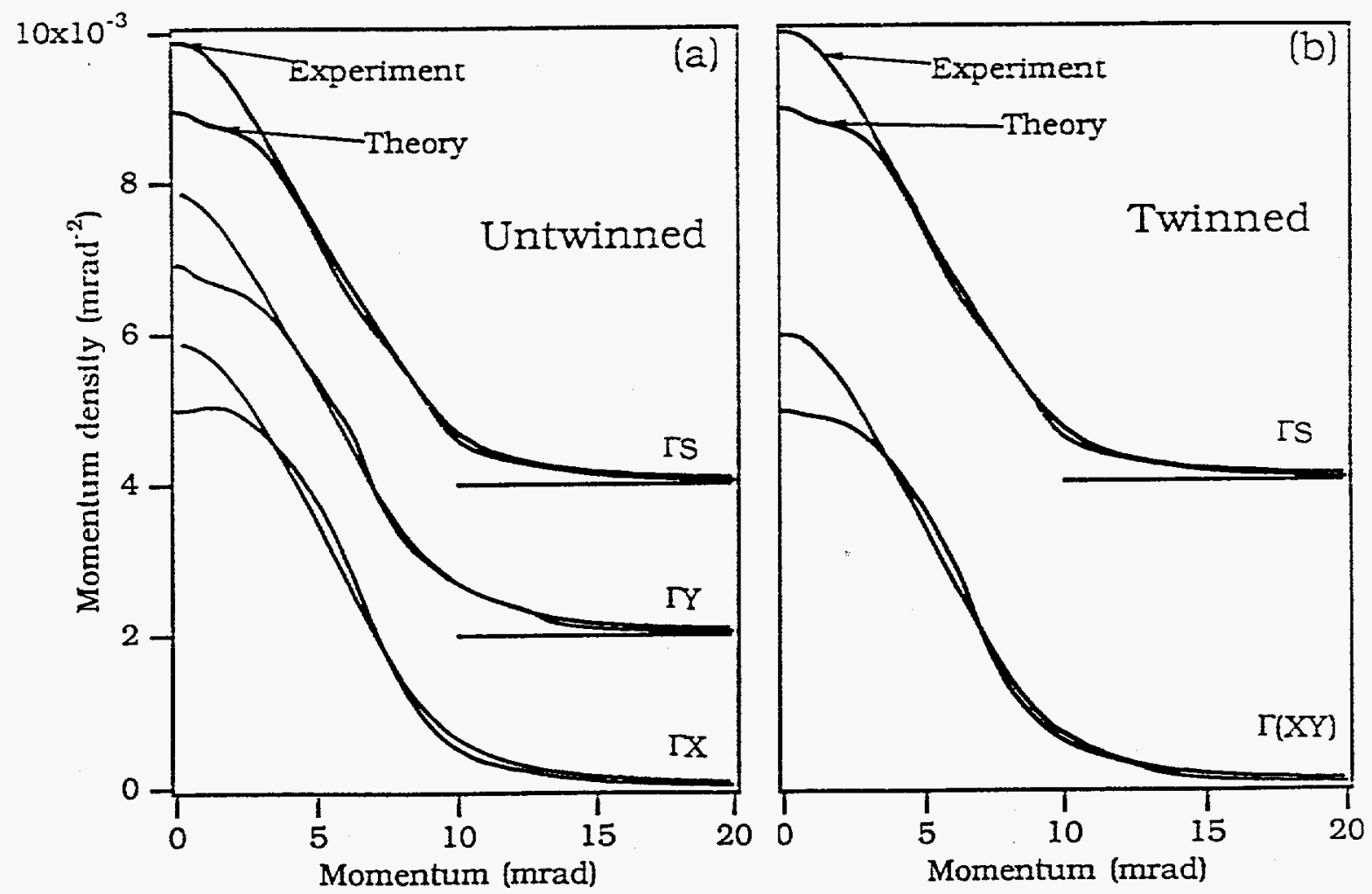

Fig. 6 Smedskjaer et al 1992 


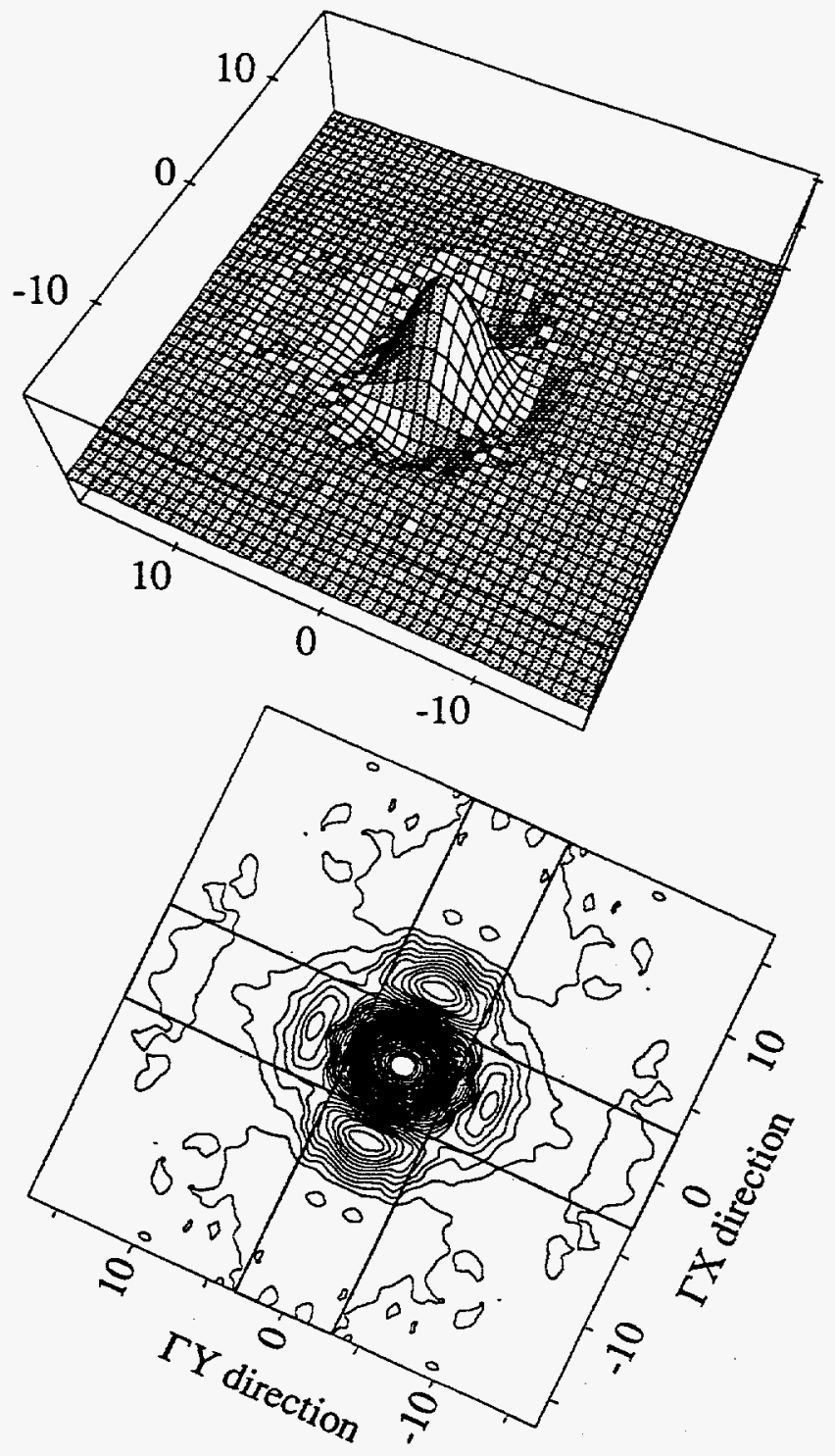

Fig. 7 Smedskjaer et al 1992 


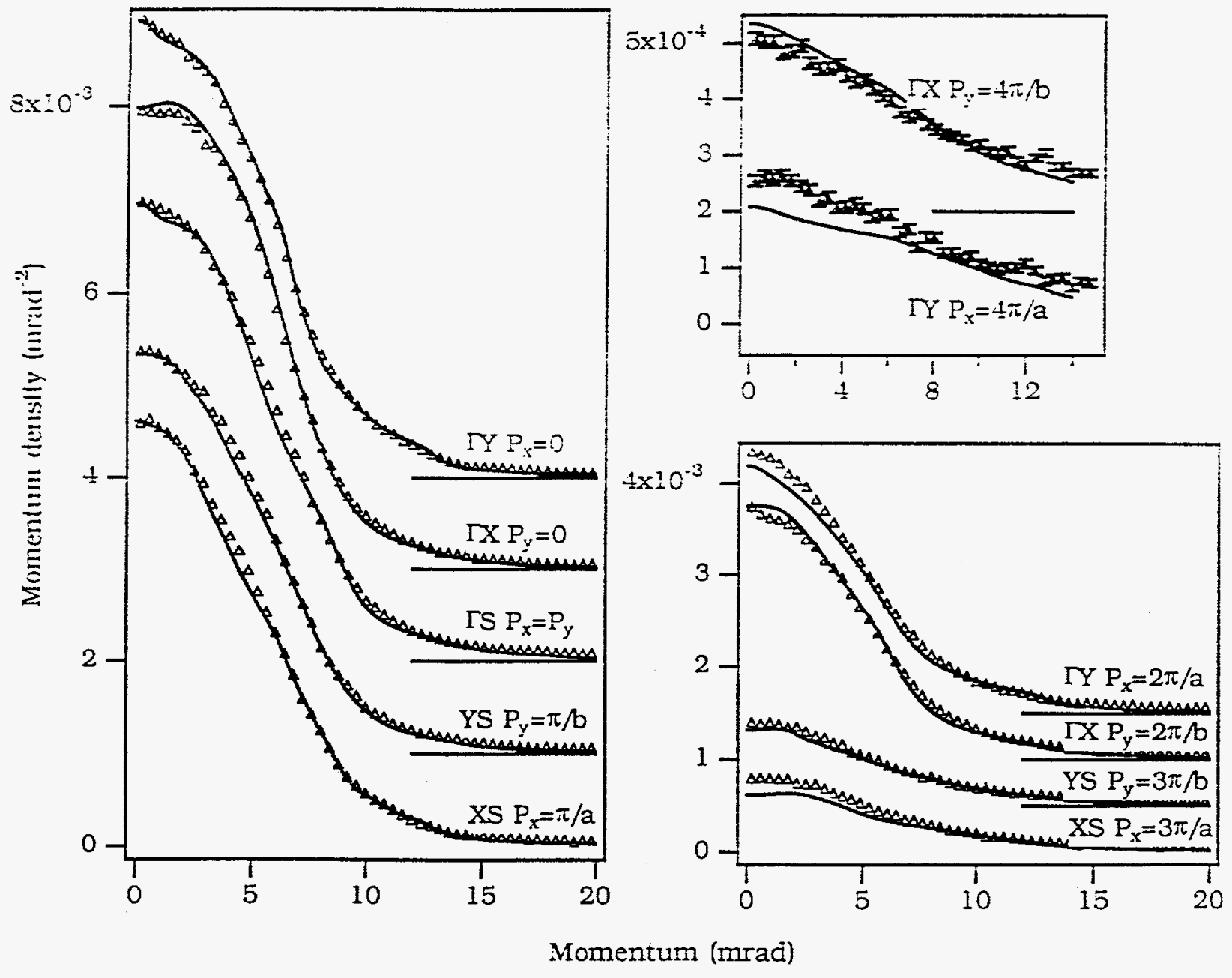

Fig. 8 Smedskjaer et al 1992 


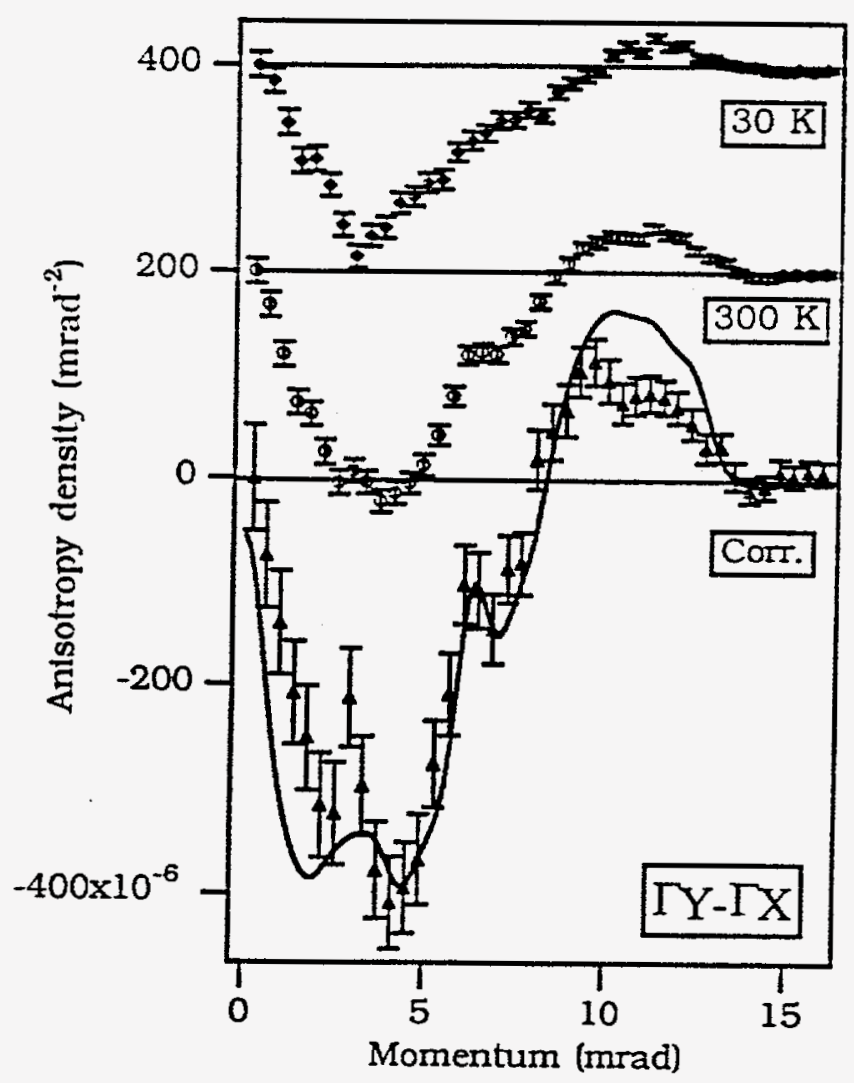

Fig. 9 Smedskjaer et al 1992 

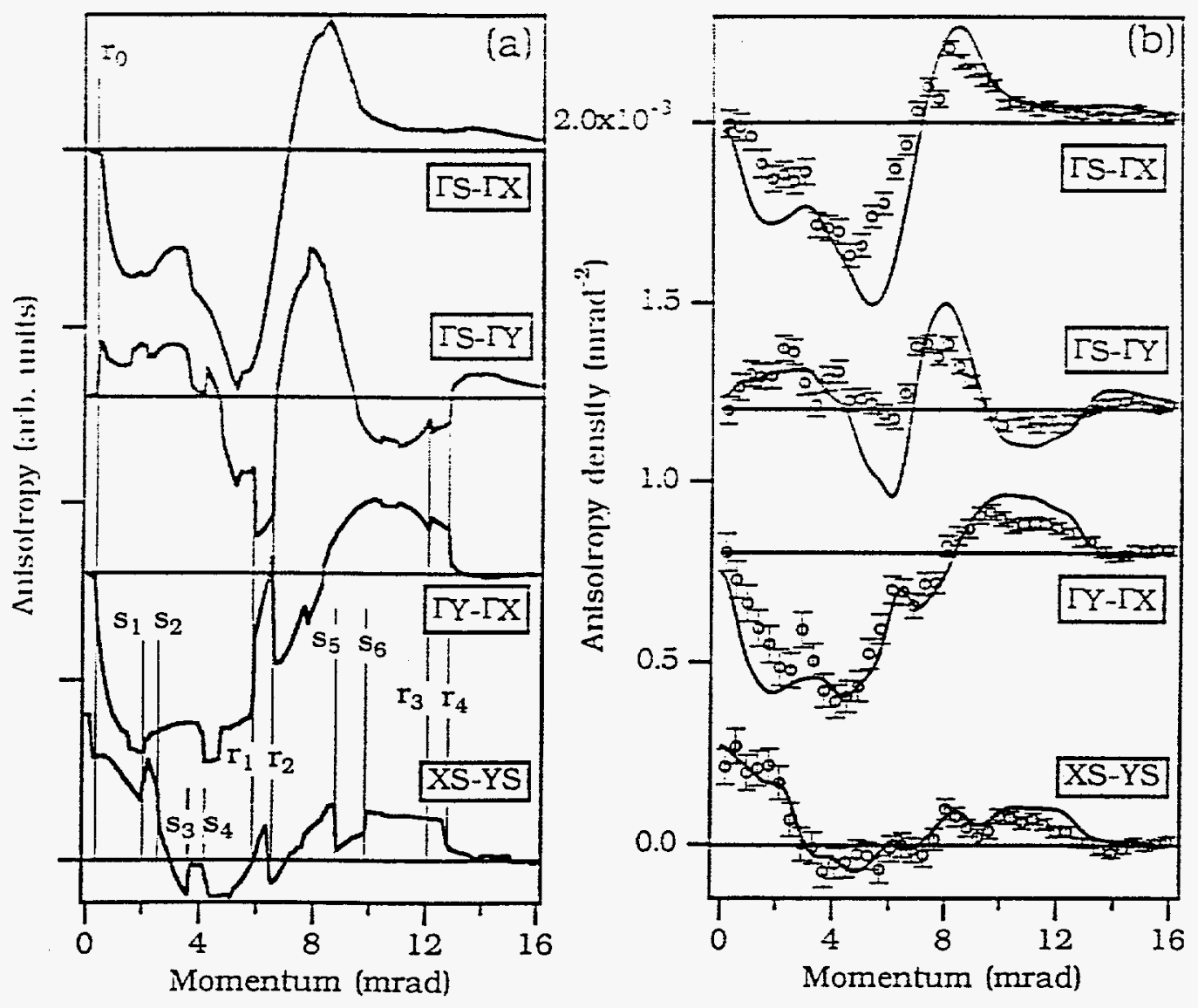

Fig. 10 Smedskjaer et al 1992 


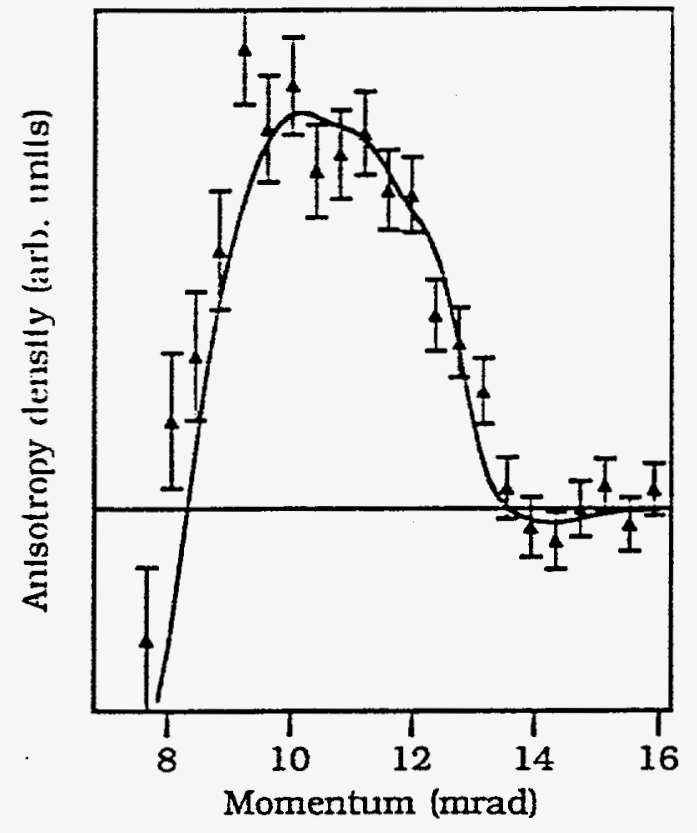

Fig. 11 Smedskjaer et al 1992 